\title{
Activation of the mTORC1 and STAT3 pathways promotes the malignant transformation of colitis in mice
}

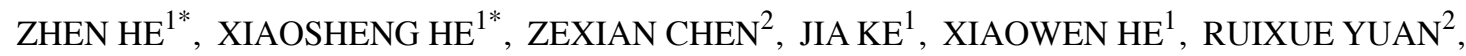 \\ ZERONG CAI ${ }^{2}$, XIUTING CHEN ${ }^{2}$, XIAOJIAN WU ${ }^{1}$ and PING LAN ${ }^{1}$ \\ ${ }^{1}$ Department of Colorectal Surgery, The Sixth Affiliated Hospital, Sun Yat-sen University, Guangzhou, Guangdong 510655; \\ ${ }^{2}$ Gastrointestinal Institute, Sun Yat-sen University, Guangzhou, Guangdong 510655, P.R. China
}

Received June 17, 2014; Accepted July 14, 2014

DOI: $10.3892 /$ or.2014.3421

\begin{abstract}
Chronic inflammation is an underlying risk factor for colorectal cancer. No direct evidence has proven that inflammation in the colon promotes carcinogenesis. STAT3 plays an important role in the development of colitis-associated colorectal cancer (CAC). There is crosstalk between the mammalian target of rapamycin complex 1 (mTORC1) and the STAT3 pathways. The aim of the present study was to confirm that colitis promotes $\mathrm{CAC}$ and if so, to explore the function of the STAT3 and mTORC1 pathways in CAC. C57BL/6 mice were treated with axozymethane (AOM) and dextran sulfate sodium (DSS) to induce CAC. By varying the concentration of DSS ( 0,1 and $2 \%$ respectively), we mimicked the CAC model with different degrees of inflammation and determined the risk of carcinogenesis. Expression of the STAT3 and mTORC1 pathways was detected. Finally, rapamycin, an mTORC1 inhibitor, was used to treat the CAC model. Tumor load, protein and gene expression of chemokines were determined. The multiplicity and tumor load of the high inflammation group were higher than those of the low inflammation group. Immunohistochemical staining and western blot analysis revealed that activation of the STAT3 and mTORC1 pathways increased gradually in the inflammation tissues and tumors. When we treated the mice with rapamycin, the tumor incidence, multiplicity and tumor load decreased. In addition, rapamycin widely suppressed the expression of pro-inflammatory and anti-inflammatory chemokines in the tissues, including tumor necrosis factor- $\alpha$, interferon- $\gamma$, IL- 6 , IL-10 and IL-12 $\alpha$. In conclusion, inflammation promotes the development of CAC
\end{abstract}

Correspondence to: Professor Xiaojian Wu or Professor Ping Lan, Department of Colorectal Surgery, The Sixth Affiliated Hospital of Sun Yat-sen University, Guangzhou, Guangdong 510655, P.R. China

E-mail: wxjmqy2003@yahoo.com

E-mail: lpzm@yahoo.com

*Contributed equally

Key words: inflammation, colitis-associated colorectal cancer, STAT3, mTORC1 via the STAT3 and mTORC1 pathways, which may be a viable treatment strategy for the chemoprevention of CAC.

\section{Introduction}

Chronic inflammation and inflammatory responses are considered to play crucial roles in the development of cancer (1). Inflammatory bowel diseases (IBDs), characterized by immune deregulation and autoimmunity, are recognized to have a high risk for the development of colitis-associated colorectal cancer (CAC) $(2,3)$. Animal models of IBDs have presented important clues to the decisive roles of inflammatory mediators and molecular events leading to the development of CAC (4). Within this population, the variability in the cancer risk is complex. Although genetic variability plays an important role in the malignant transformation of IBDs (5), the long duration of the disease, as well as the severity and extent of the inflammation are considered to be the most important risk factors, particularly the severity of inflammation (6). However, changes at the molecular level under different degrees of inflammation in colitis and CAC still remain puzzling.

mTOR, a serine/threonine kinase that regulates cell cycle progression, cellular proliferation and growth, autophagy and angiogenesis, is often deregulated in human cancers $(7,8)$. Functioning as the protein kinase of the mammalian target of rapamycin complex 1 (mTORC1), mTOR regulates the regulators of eukaryotic translation initiation factor $4 \mathrm{E}$ binding protein 1 (4EBP1) and the p70 ribosomal protein S6 kinase 1 (S6K1) $(9,10)$. Activation of mTORC1 actuates protein synthesis via activation of S6K1, which phosphorylates the ribosomal protein S6 and initiates translation (11). S6K1, a member of the AGC kinase family, plays important roles in cell growth, proliferation and cell differentiation via a wide range of extracellular signals including growth factors, hormones, nutrients and stress $(12,13)$. Rapamycin and its analogs are likely to be the first mTOR-perturbing drugs for therapeutic use in human cancer (14). Rapamycin is the universal inhibitor of mTORC1 and S6K1 and is a cell-type specific inhibitor of mTORC2 and Akt signaling (15). Various tumor cells have been effectively treated with rapamycin, including rhabdomyosarcoma, osteosarcoma, pancreatic carcinoma, RCC, Ewing sarcoma, brain, lung, prostate and breast cancer (16). Intriguingly, Thiem et al found that coactivation of mTOR and STAT3 signaling occurs 
in gastric tumors in humans. They demonstrated that mTORC1 is activated via GP130 in a STAT3- and STAT1-independent manner and induces inflammation-associated gastrointestinal tumorigenesis (17). Generally, excessive activation of STAT3 has been identified as a hallmark of inflammation-associated cancers (18). STAT3 activation commonly contributes to an abundance of tumor and stromal cell-derived cytokines, such as IL-6 and IL-11 (19). It has been reported that IL-6dependent persistent activity of STAT3 is required to maintain the survival capacity of intestinal epithelial cells and the development of colitis-associated cancer (19). However, the relationship between the activation of STAT3 and mTORC1 in colitis and CAC is not yet clear.

In the present study, we employed the colonotropic mutagen azoxymethane (AOM) and different concentrations of dextran sulfate sodium salt (DSS) to produce different degrees of inflammation in the CAC mouse model. We determined the activity of mTOR and STAT3 and compared the efficiency of tumor formation at the different inflammation levels. We found that as the inflammation was aggravated, the activity of STAT3 and mTOR was elevated. Coactivation of STAT3 and mTOR signaling occurred with increased inflammation and persisted until tumor development. We also demonstrated that rapamycin did not only inhibit mTOR signaling but also STAT3 signaling in colitis and suppressed inflammationassociated colon cancer.

\section{Materials and methods}

Animals. All female mice (C57BL/6) used were 7-8 weeks of age, with body weight 18-20 g. They were housed in pathogen-free rooms in filter-topped cages at the Laboratory Animal Facility at Zhongshan School of Medicine, Sun Yat-sen University, Guangzhou. All mice were quarantined for 3 days after arriving. They were maintained in a room with controlled temperature $\left(22 \pm 1^{\circ} \mathrm{C}\right)$, humidity $(50-70 \%)$ and a 12-h light/dark cycle. All animal experiments were approved by the Ethics Committee for Animal Care and Use of Zhongshan School of Medicine, Sun Yat-sen University according to an approved protocol.

AOM/DSS experimental inflammation-induced CAC model. We followed a modified protocol outlined recently by Chumanevich et al (20). The CAC model was induced by AOM (Sigma-Aldrich, St. Louis, MO, USA) and DSS (MP Biomedicals, Solon, OH, USA). Briefly, female mice were injected intraperitoneally with a single dose of $\mathrm{AOM}(10 \mathrm{mg} / \mathrm{kg})$, and the mice received a course of $1-2 \%$ DSS in sterile drinking water for 7 days followed by pure drinking water for 14 days in a total of 3 cycles. Assessment of AOM/DSS-treated mice was performed daily for general appearance, food uptake, body weight, stool consistency and rectal bleeding. In the control group, the same procedure was performed with an intraperitoneal injection of $\mathrm{AOM}(10 \mathrm{mg} / \mathrm{kg})$ and drinking pure water instead of DSS. A scheme of the treatment protocol is shown in Fig. 1.

Before AOM injection, the mice were randomized into control and experimental groups (20 animals per group). Rapamycin (LC Laboratories, Woburn, MA, USA) was dissolved in ethanol and stored at $-80^{\circ} \mathrm{C}$. For dosing of animals, the stock solution was diluted with aqueous solution to give final concentrations of $4 \%$ ethanol, $5 \%$ polyethylene glycol 400 and 5\% Tween-80 immediately before intraperitoneal injections (21). After 14 days of AOM injection, mice in the experimental group received $0.75 \mathrm{mg} / \mathrm{kg}$ rapamycin every day and the control animals received the drug vehicle only according to the same dosing schedule. The mice were sacrificed 63 days after AOM injection. A scheme of the treatment protocol is shown in Fig. 2. The mouse colons were isolated, slit open longitudinally for tumor count and size determination using a dissecting microscope. Tumors were excised for RNA and protein isolation for gene expression analysis using real-time PCR and western blot analysis, respectively. Non-tumorous colonic epithelia were scraped from the middle and proximal portions of the colon. Colons adjacent to tumors were fixed in $10 \%$ fresh formalin, and sections were cut out to embed in paraffin for immunohistochemical analysis.

$m R N A$ isolation and quantitative expression studies. Total RNA was isolated from the AOM/DSS + rapamycin and AOM/DSS + vehicle groups using the AxyPrep ${ }^{\text {TM }}$ Multisource Total RNA Miniprep kit (Axygen, Union City, CA, USA) according to the manufacturer's suggested protocol. Total RNA $(0.5 \mu \mathrm{g})$ was reverse transcribed using ReverTra Ace ${ }^{\circledR}$ qPCR RT kit (Toyobo, Osaka, Japan) in a $20-\mu 1$ reaction volume with the GeneAmp PCR System 9700 (Applied Biosystems, Foster City, CA, USA) and from that, $2 \mu \mathrm{l}$ of the reaction volume was added to Thunderbird SYBR qPCR Mix (Toyobo) for quantitative real-time PCR using the 7500 Real-Time PCR System (Applied Biosystems). Primers used were as follows: forward (GCCAGCCGATGGGTTGTAC) and reverse (TTGACGG CAGAGAGGAGGTT) primers for the detection of TNF- $\alpha$ expression; forward (TTACTGCCACGGCACAGTCA) and reverse (TGCAGGATTTTCATGTCACCAT) primers for IFN- $\gamma$ expression; forward (AACCACGGCCTTCCCTACTT) and reverse (TCTTTTCTCATTTCCACGATTTCC) primers for IL-6 expression; forward (CAGGGCCCTTTGCTATGGT) and reverse (TCTGAGCTGCTGCAGGAATG) primers for IL-10 expression; forward (GCCATCAACGCAGCACTTC) and reverse (TGCTTCTCCCACAGGAGGTT) primers for IL-12 $\alpha$ expression and actin was chosen as a housekeeping gene with forward (ATGACCCAAGCCGAGAAGG) and reverse (CGGCCAAGTCTTAGAGTTGTTG) primers. Expression of various genes was normalized to the expression of actin.

Histological analysis of the colonic tumors in the AOM/DSS-treated mice. Mouse colon tissues were fixed in 4\% paraformaldehyde, paraffin-embedded and sectioned at a $4-\mu \mathrm{m}$ thickness. The sections were stained with hematoxylin and eosin (H\&E) and microscopically examined for histopathologic and inflammatory changes as described by Chumanevich et al (20).

Immunohistochemistry and western blot analysis. Standard immunohistochemistry and western blotting techniques were used. The antibodies used were as follows: mTOR was detected with polyclonal anti-rabbit mTOR antibody [Abcam (Hong Kong) Ltd., HKSP, New Territories, Hong Kong]; phosphorylated (p)-mTOR (S2448) was detected with 


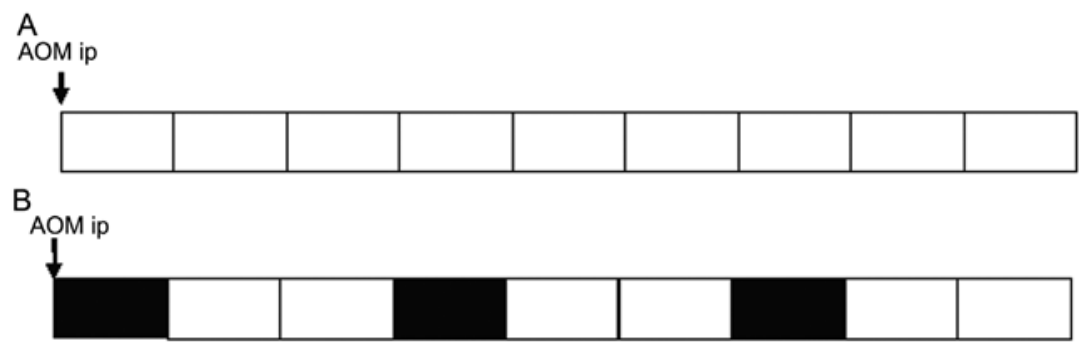

Figure 1. Experimental protocol of the CAC model. Treatment was as follows: (A) control group, the mice were intraperitoneally (ip) injected with AOM and received pure water for 9 weeks; (B) experimental group, the mice were ip injected with AOM and received 1-2\% DSS for 1 week, followed by 2 weeks of pure water in a total of 3 cycles ( $n=6$ per group). CAC, colitis-associated colorectal cancer; AOM, axozymethane; DSS, dextran sulfate sodium.

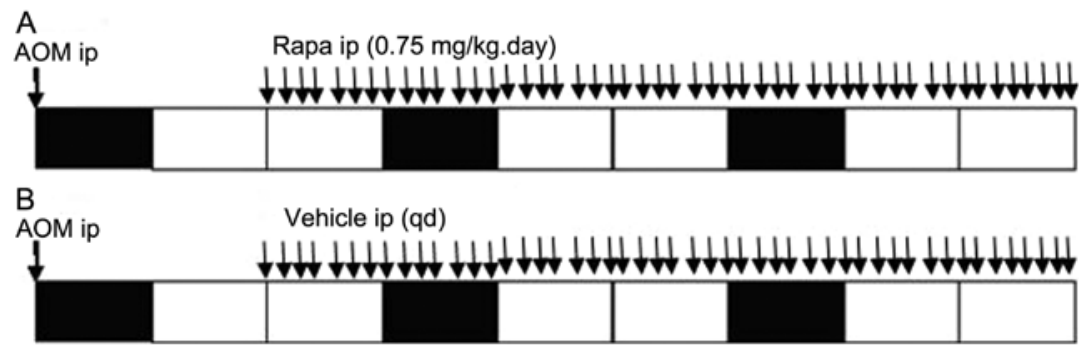

Figure 2. Experimental protocol of the rapamycin-treated CAC model. Treatment was as follows: (A) experimental group, the mice were intraperitoneally (ip) injected with rapamycin (Rapa) $0.75 \mathrm{mg} / \mathrm{kg}$ from day 14 to day 63 daily; (B) control group, the mice were ip injected with vehicle from day 14 to day 63 once daily (qd) ( $\mathrm{n}=20$ per group). CAC, colitis-associated colorectal cancer.

polyclonal anti-rabbit p-mTOR (S2448) antibody (Abcam); S6K was detected with monoclonal anti-rabbit S6K antibody (Abcam); p-S6K (T389) was detected with monoclonal antirabbit p-S6K (T389) antibody (Abcam); STAT3 was detected with monoclonal anti-rabbit STAT3 antibody (Cell Signaling Technology, Danvers, MA, USA); p-STAT3 (Tyr705) was detected with monoclonal anti-rabbit p-STAT3 (Tyr705) antibody (Cell Signaling Technology); GAPDH was detected with polyclonal anti-GAPDH (Santa Cruz Biotechnology, Santa Cruz, CA, USA).

Statistical analysis. Data are expressed as the mean \pm standard error of the mean (SEM). All statistical analyses were carried out using an unpaired t-test analysis in GraphPad Prism 4 software (GraphPad Software, San Diego, CA, USA). Significance was defined by $\mathrm{P}<0.05$.

\section{Results}

Inflammation promotes the malignant transformation of colitis in an experimental colitis-associated colorectal cancer $(C A C)$ model. To imitate different levels of colonic inflammation, the concentration of dextran sulfate sodium (DSS) in drinking water was changed. We randomized mice into 3 groups (6 animals per group) and administered axozymethane (AOM) only to the control group, $1 \%$ DSS + AOM to the low inflammation group and 2\% DSS + AOM to the high inflammation group. This showed that a higher concentration of DSS in drinking water could induce higher colonic inflammation. A lower concentration of DSS (1\% DSS + AOM) had less effect on body weight loss when compared with the control group (AOM only); mice in the high inflammation group (2\% DSS + AOM) showed a higher body weight loss when compared with the mice in the control group (AOM only) and the low inflammation group (1\% DSS + AOM) (Fig. 3A). The colon length was significantly reduced in the high inflammation group than that in the low inflammation group (Fig. 3B). Specifically, high inflammation shrank the colon to $58.9 \pm 7.1 \mathrm{~mm}(\mathrm{P}=0.001$ vs. AOM only) and lower inflammation shrank the colon to $67.6 \pm 5.2 \mathrm{~mm}(\mathrm{P}=0.01 \mathrm{vs}$. AOM only). The high inflammation group had shorter colons than those of the low inflammation group $(\mathrm{P}=0.044)$. In addition, the histopathological inflammation score in the high inflammation group was significantly higher than that in the low inflammation group $(3.3 \pm 1.0$ vs. 2.0 $\pm 1.2, \mathrm{P}=0.016$ ) (Fig. 3C). Single intraperitoneal injection of the mutagenic agent $\mathrm{AOM}$ and repeated oral administration of the pro-inflammatory agent DSS could induce the CAC model (Fig. 3D). The tumor multiplicity of the high inflammation group was higher than that of the low inflammation group (5.2 \pm 0.9 vs. $2.2 \pm 0.7, \mathrm{P}=0.0232$ ) (Fig. 3E), and the tumor load (sum of tumor diameters per mouse) in these two groups was also significant $(19.4 \pm 2.0$ vs. $4.7 \pm 1.6, \mathrm{P}=0.0002)$ (Fig. 3F).

STAT3 and mTORC1 pathways are highly activated in colitis-associated cancer. It has been shown that the STAT3 and mTORC1 pathways may play an important role in inflammation and carcinogenesis $(7,22)$. To illuminate the function of the STAT3 and mTORC1 pathways in CAC, we determined the expression of STAT3 and mTORC1 pathways in colonic tumors and inflammatory colonic tissues adjacent to tumors. Phosphorylated (p)-STAT3 (Tyr705), the active phase of STAT3, in the high inflammation colon tissue was expressed at a higher level than that in the low inflammation tissues while the expression of total STAT3 was not 
A

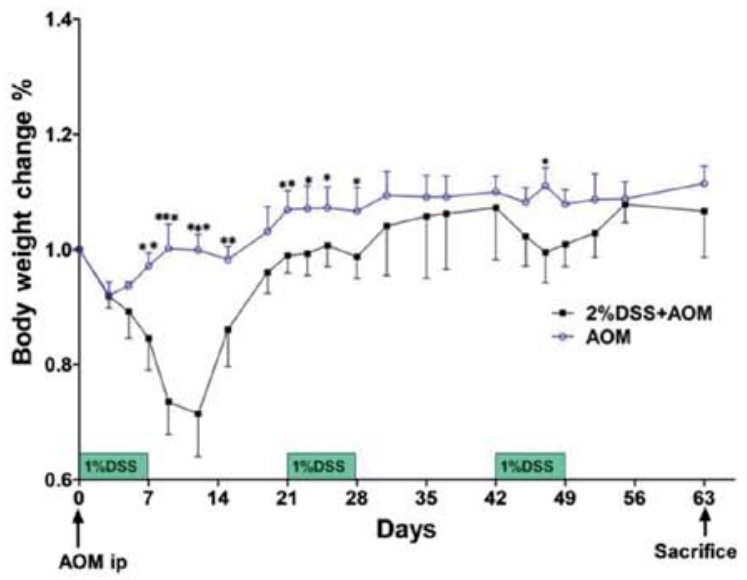

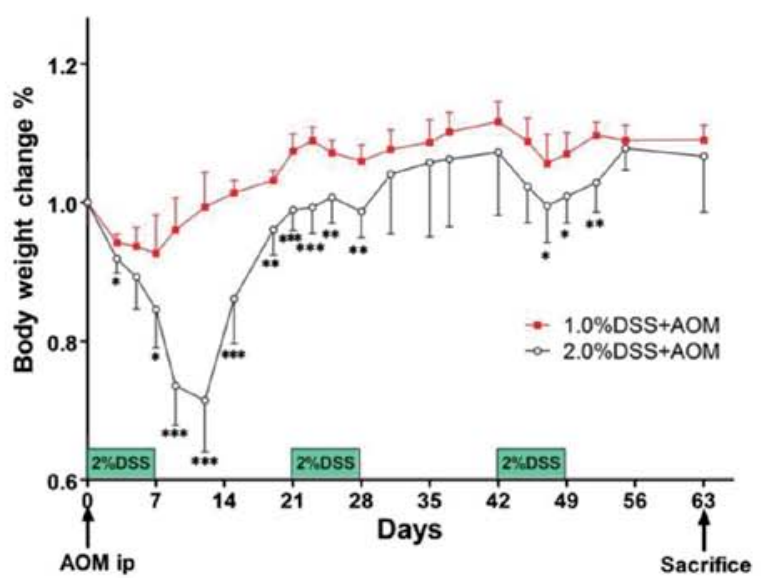

C

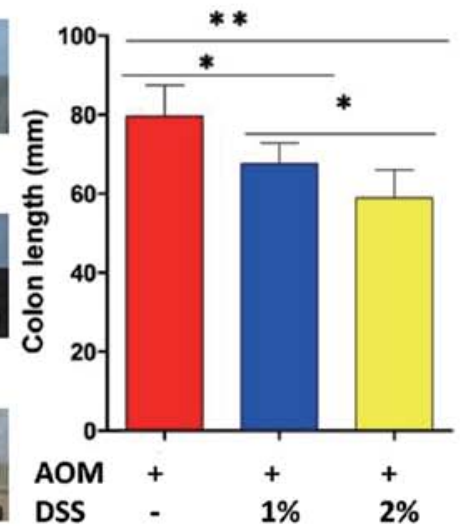

$\mathbf{E}$

$\mathbf{F}$

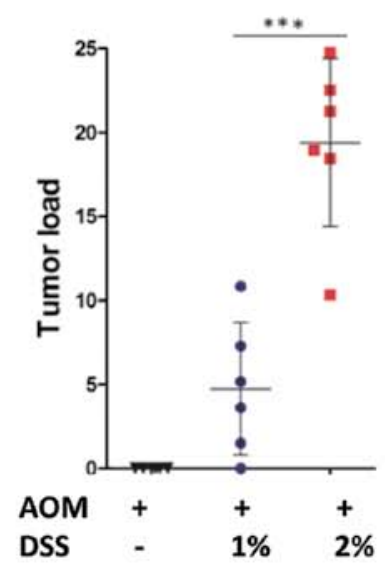

Figure 3. Inflammation promotes the development of CAC. Female C57BL/6 mice were randomized into 3 groups (6 animals per group): control group (AOM only), low inflammation group (AOM + 1\% DSS) and high inflammation group (AOM + 2\% DSS). (A) Body weight change. (B) Colon length change. (C) Histological scoring of inflammation. (D) Macroscopic and microscopic (H\&E staining; magnification, x40) views of the colonic tumors. (E) Number of colonic tumors per animal (multiplicity) and (F) tumor load (sum of the tumor diameters per mouse) per animal. ${ }^{*} \mathrm{P}<0.05,{ }^{* *} \mathrm{P}<0.01,{ }^{* * *} \mathrm{P}<0.001$. CAC, colitisassociated colorectal cancer; AOM, axozymethane; DSS, dextran sulfate sodium.

obviously altered (Fig. 4A). Moreover, the expression of p-STAT3 in the colitis-associated cancer was higher than that in the high inflammation tissues (Fig. 4B), indicating that with the development of inflammation, the activation of the STAT3 signaling pathway increased gradually. p-mTOR (S2448) is one of the active phases of mTOR and as one of 

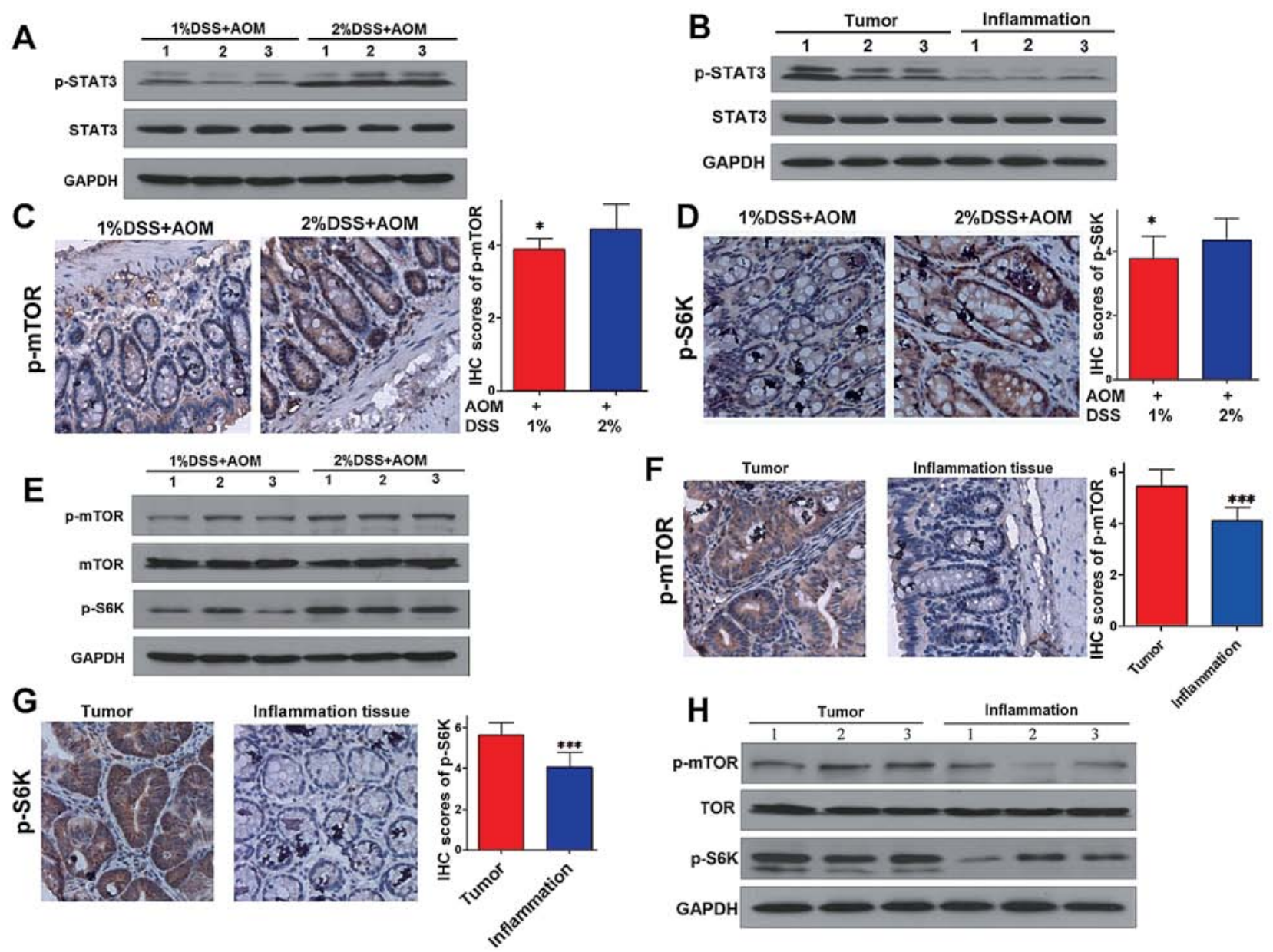

Figure 4. STAT3 and mTORC1 pathways are highly activated in CAC. Immunohistochemical analysis of low inflammation tissues, high inflammation tissues and tumors for phosphorylated (p)-mTOR (S2448) and p-S6K (T389). Western blot analysis of low inflammation tissues, high inflammation tissues and tumors for STAT3, p-STAT3 (Tyr705), mTOR, p-mTOR (S2448), p-S6K (T389) and glyceraldehyde 3-phosphate dehydrogenase (GAPDH) housekeeping gene. (A and B) Expression of the STAT3 pathway in the low inflammation tissues, high inflammation tissues and tumors. (C) Magnification, x200, (D) magnification, x200, and (E) expression of the mTORC1 pathway in the low inflammation tissue and higher inflammation tissue. (F) Magnification, x200, (G) magnification, x200, and $(\mathrm{H})$ expression of the mTORC1 pathway in high inflammation tissues and tumors. ${ }^{*} \mathrm{P}<0.05,{ }^{* *} \mathrm{P}<0.01,{ }^{* * * *} \mathrm{P}<0.001$. CAC, colitis-associated colorectal cancer.

the downstream molecules of the mTORC1 pathway, the active phase of S6K is phosphorylated-S6K (T389). Fig. 4C-E shows that the expression levels of p-mTOR (S2448) and p-S6K (T389) in the high inflammation tissues were higher than these levels in the low inflammation tissues. As shown in Fig. 4F-H, the expression levels of p-mTOR (S2448) and p-S6K (T389) in colitis-associated cancer were higher than these levels in the high inflammation tissues. This indicated that with the development of inflammation, the activation of the mTORC1 pathway increased gradually similar to the STAT3 pathway.

mTORC1 inhibitor suppresses the malignant transformation of colitis in mice. To further illuminate the role of the mTORC1 and STAT3 pathways in CAC, we used an mTORC1 inhibitor, rapamycin, to block the mTORC1 pathway in vivo. After 14 days of AOM injection, mice in the experimental group were peritoneally injected with $0.75 \mathrm{mg} / \mathrm{kg}$ rapamycin daily, and the control animals received drug vehicle only according to the same dosing schedule (20 animals per group) (Fig. 2). After 63 days of AOM injection, all mice were sacrificed, and the tumor incidence, multiplicity and tumor size were evaluated.
Rapamycin, the mTORC1 inhibitor, reduced the incidence of CAC to $40 \%(8 / 20)$, compared with $100 \%(20 / 20)$ in the control group (vehicle + AOM/DSS $)(P<0.001)$. The multiplicity of CAC in the experimental group was significantly lower than that in the control group $(1.55 \pm 1.92$ vs. $8.30 \pm 3.59$, $\mathrm{P}<0.001$ ) (Fig. 5A and B). In addition, the tumor load (sum of tumor diameters per mouse) in these two groups was significant $(4.8 \pm 2.3$ vs. $18.1 \pm 8.0, \mathrm{P}<0.001)$ (Fig. $5 \mathrm{C})$.

\section{Rapamycin suppresses the malignant transformation through} inhibition of the MTORCI and STAT3 pathways. To confirm the effect of rapamycin on the mTORC1 and STAT3 pathways, we detected the expression of mTORC1 and STAT3 pathways in colon tissues by western blotting. Previous research showed that there exists crosstalk between the mTORC1 and STAT3 pathway in vitro (23). In the present study, as shown in Fig. 5D, the mTORC1 inhibitor rapamycin reduced the expression of p-STAT3 (Tyr705), which indicated that rapamycin inhibited the activation of the STAT3 pathway in vivo. Moreover, rapamycin also inhibited the activation of the mTORC1 pathway in vivo (Fig. 5D). 
A

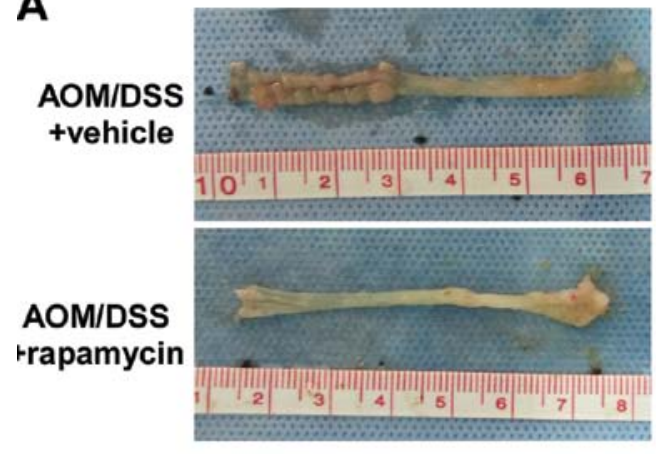

D

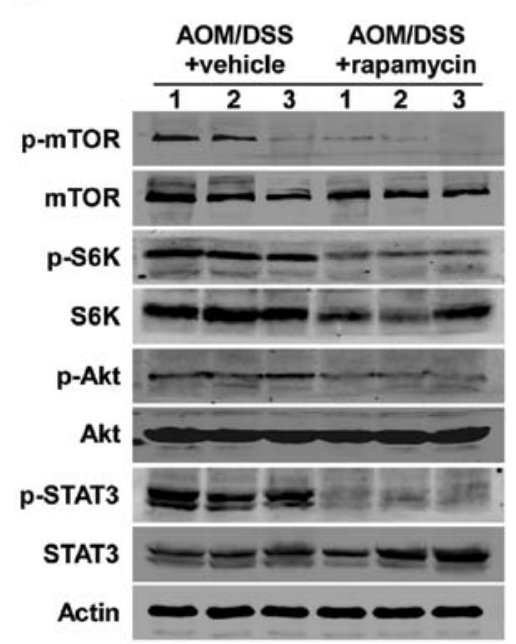

B

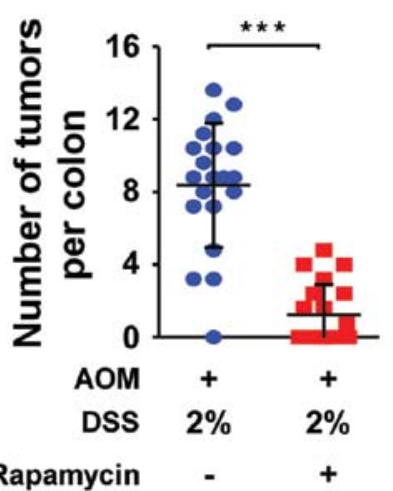

Rapamycin

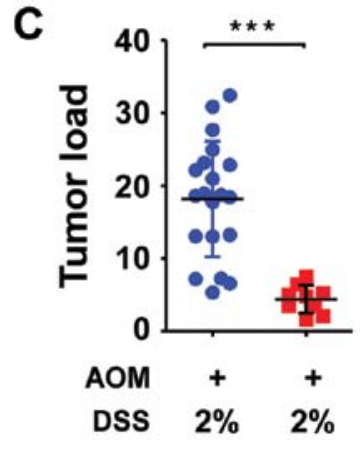

Rapamycin - +

E

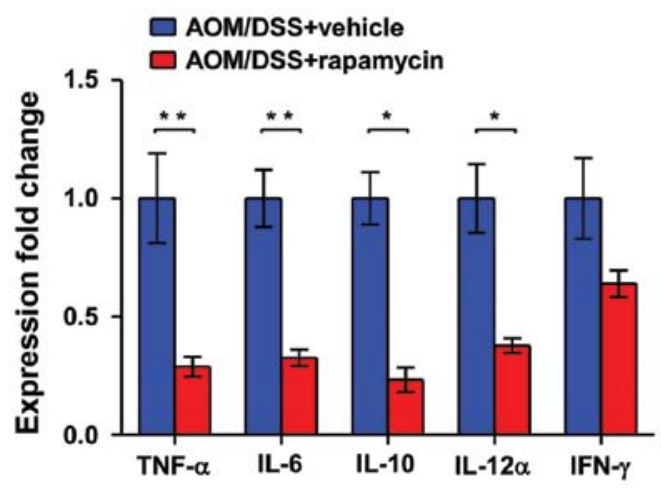

Figure 5. Rapamycin, an inhibitor of the mTORC1 pathway, inhibits the development of CAC and reduces the expression of pro-inflammatory and antiinflammatory chemokines. (A) Macroscopic view of colons of CAC treated with rapamycin. (B and C) Number of colonic tumors per animal (multiplicity) and tumor load (sum of tumor diameters per mouse) per animal $(\mathrm{n}=20)$ following treatment with rapamycin. (D) Western blot analysis of the colon tissues for the STAT3 and mTORC1 pathways (n=3 per group). (E) Effects of rapamycin on colonic TNF- $\alpha$, IL-6, IL-12 $\alpha$, IL-10 and INF- $\gamma$ mRNA expression patterns Both the experimental group (AOM/DSS + rapamycin) and control group (AOM/DSS + vehicle) mice were sacrificed on day 63 following 3 cycles of $2 \%$ DSS administration. Following sacrifice, the expression levels of TNF- $\alpha$, IL-6, IL-12 $\alpha$, IL-10 and INF- $\gamma$ were quantified by TaqMan-qPCR, relative to the expression of the housekeeping gene (actin). mRNA expression for each gene was quantified for each individual mouse in triplets and averaged per mouse ( $\mathrm{n}=7$ per group). Data represent mean $\pm \mathrm{SEM} ;{ }^{*} \mathrm{P}<0.05,{ }^{* *} \mathrm{P}<0.01,{ }^{* * *} \mathrm{P}<0.001 . \mathrm{n}=7$ in each group. CAC, colitis-associated colorectal cancer.

Rapamycin reduces the expression of pro-inflammatory and anti-inflammatory chemokines. As demonstrated above, inflammation is one of the main processes driving tumor progression in CAC. Therefore, to direct our mechanistic study of how rapamycin, an mTORC1 inhibitor, reduced $\mathrm{CAC}$, changes in colonic messenger RNA expression levels of common pro-inflammatory and anti-inflammatory cytokines were determined. Compared with mice in the control group, rapamycin suppressed the colonic mRNA expression of proinflammatory cytokines, such as tumor necrosis factor- $\alpha$ $(\mathrm{TNF}-\alpha)(\mathrm{P}=0.002)$, IL-6 $(\mathrm{P}<0.001)$ and IL-12 $\alpha(\mathrm{P}=0.01)$. Notably, rapamycin also suppressed the colonic mRNA expression of anti-inflammatory cytokine IL-10 $(\mathrm{P}<0.001)$. Furthermore, mRNA expression of another anti-inflammatory cytokine, IFN- $\gamma$, in colonic tissues of rapamycin-treated CAC mice tended to be reduced $(\mathrm{P}=0.179$, Fig. $5 \mathrm{E})$.

\section{Discussion}

Recent research has demonstrated that the risk factors for the development of colorectal neoplasia in patients with IBD include disease duration, anatomic extent of the disease, age at onset of IBD, family history of sporadic CRC and severity of inflammation (24). Among these factors, severity of inflammation may be the most crucial and the easiest to remedy. A cohort study showed that the severity of microscopic inflammation over time is an independent risk factor for developing advanced colorectal neoplasia among patients with longstanding ulcerative colitis (25). In addition, in Crohn's disease patients, perianal disease, bypasses and strictures may be sites of increased risk for neoplastic transformation $(26,27)$. These findings indicate that, in IBD patients, chronic intestinal inflammation may be the leading cause of CAC. Moreover, a large epidemiological study showed that patients regularly ingesting anti-inflammation agent, aminosalicylates (5-ASA) for IBD treatment had a lower CRC risk $(28,29)$. These findings together strongly indicate that inflammation plays an important role in CAC development. In the present study, we varied the concentration of pro-inflammatory agent, DSS, in drinking water to induce different degrees of colonic inflammation. We found that the tumor multiplicity in mice in the high inflammation group was higher than that in the low inflammation group, 
which was consistent with the colonic inflammatory activity. In addition, tumor size in the high inflammation group tended to be larger. All of these findings directly demonstrate that inflammation promotes the malignant transformation of colitis.

STAT3 belongs to the STAT (signal transducer and activator of transcription) family of signal responsive transcription factors, which similar to $\mathrm{NF}-\kappa \mathrm{B}$ are kept in an inactive form in the cytoplasm of non-stimulated cells (18). Activation of STAT3 is mediated by phosphorylation of a tyrosine residue (Tyr705) that induces STAT3 dimerization through phosphotyrosine-SH2 domain interaction. Activators of STAT3 include interleukins, epidermal growth factor (EGF) family members and interferon (18). When activated in cancer or immune cells, STAT3 can induce the expression of a wide variety of genes including IL-6, IL-22, EGF, IL-23 and IL-10 as well as protooncogenes such as K-Ras, Src and c-Abl, whose products are capable of inducing STAT3 activation in return (22). Therefore, there is no doubt that STAT3 is a crucial link between inflammation and cancer. Grivennikov et al recently demonstrated that ablation of IL-6 reduced the activation of STAT3, which was consistent with the reduction in CAC tumorigenesis. When STAT3 in IL-6 ${ }^{-/}$animals was conditionally knocked out, there was almost no tumorigenesis in the CAC model (19). In the present study, the activation of the STAT3 signaling pathway was more excessive in the high inflammation tissues than that in the low inflammation tissues. Moreover, the activation of the STAT3 signaling pathway was higher in the tumors than that in the inflammation tissues. These findings suggest that with the development of inflammation, the activation of the STAT3 pathway increased gradually. Moreover, CAC carcinogenesis was associated with the persistent activation of the STAT3 pathway.

The mTORC1 pathway integrating both intracellular and extracellular signals, serves as a central regulator of cell metabolism, growth, proliferation and survival. mTORC1 is also a crucial molecule in the processes of inflammation and cancer development. Dysregulation of the mTORC1 pathway is commonly found in human inflammation and cancers (30-33). Rapamycin, an inhibitor of mTORC1, was originally developed as an immunosuppressive agent, suggesting that the mTORC1 pathway plays an important role in the regulation of the immune system. mTORC1 is essential for survival, cytokine production and migration of immune cells involved in innate immune response and adaptive immune response, such as mast cells (34), macrophages (35), natural killer cells (36), B cells (37) and T cells (38), which play a crucial role in the inflammatory response. Furthermore, the mTORC1 signaling pathway also plays an important role in carcinogenesis. Dysregulation of the mTORC1 pathway can lead to many types of cancers, such as lung (39) and breast (31), hepatocarcinoma (33) and colorectal cancer (32). Foremost, there exists crosstalk between the mTOR and the STAT3 signaling pathway which is a crucial pathway in CAC as mentioned above. A recent study showed that when human breast, prostate, lung, pancreatic and liver cancer cell lines were treated with rapamycin, activation of the STAT3 signaling pathway decreased (40). When the mTORC1 signaling pathway was overactive using siRNA for PTEN, an inhibitor of the mTORC1 pathway, activation of the STAT3 pathway was increased (40). Therefore, we hypothesized that the mTORC1 pathway may play an important role in CAC. Farkas et al recently showed that rapamycin, an mTORC1 inhibitor, ameliorated experimental murine colitis (41). Collectively, these findings demonstrate that mTORC1 may be a therapeutic target for IBD and CAC. In the present study, we found that activation of the mTORC1 pathway was gradually increased in inflammatory colic and CAC tissues. These findings indicated that with the development of inflammation, the activation of the mTORC1 signaling pathway increased gradually. CAC carcinogenesis was associated with the persistent activation of the mTORC1 pathway. When we treated the mice with rapamycin, activation of the STAT3 signaling pathway was decreased, which was consistent with previous research (42). Furthermore, the tumor incidence, multiplicity and size decreased. These findings revealed that the mTORC1 and STAT3 signaling pathways promoted the malignant transformation of colitis.

TNF- $\alpha$, IL- 6 and IL-12 are crucial pro-inflammatory cytokines in the pathogenesis of IBD. As essential switches between inflammation and cancer, much evidence has pointed to a critical role of TNF- $\alpha$, IL- 6 and IL-12 in tumor initiation, proliferation, migration, invasion and metastasis (43-46). In our study, we demonstrated that levels of mRNA expression of TNF- $\alpha$, IL-6, IL- $12 \alpha$ in mice in the CAC model treated with rapamycin were lower when compared with the levels in mice given vehicle alone. These findings reveal that rapamycin can reduce a pro-inflammatory response, which may lead to suppression of carcinogenesis. Notably, rapamycin also inhibited the expression of the anti-inflammatory cytokines, IFN- $\gamma$ and IL-10. The most viable explanation is that the mTORC1 pathway is an essential signal of immune cells such as neutrophils, monocytes, dendritic cells, B and T cells $(10,38)$. Rapamycin, an inhibitor of the mTORC1 pathway can widely suppress the expression of not only pro-inflammatory cytokines, but also anti-inflammatory cytokines.

In conclusion, the results of this study suggest that inflammation promotes colitis-associated cancer through activation of the mTORC1 and STAT3 pathways. Our data also indicate that rapamycin, an inhibitor of the mTORC1 pathway, can inhibit the development of CAC through widely suppressing the expression of pro-inflammatory and anti-inflammatory cytokines including TNF- $\alpha$, IL-6, IL-12 $\alpha$, IL-10 and INF- $\gamma$.

\section{Acknowledgements}

This study was supported by grants from the National Natural Science Foundation of China (nos. 81072046 and 91029702).

\section{References}

1. Balkwill $F$ and Mantovani A: Inflammation and cancer: back to Virchow? Lancet 357: 539-545, 2001.

2. Eaden JA, Abrams KR and Mayberry JF: The risk of colorectal cancer in ulcerative colitis: a meta-analysis. Gut 48: 526-535, 2001.

3. Gillen CD, Walmsley RS, Prior P, Andrews HA and Allan RN: Ulcerative colitis and Crohn's disease: a comparison of the colorectal cancer risk in extensive colitis. Gut 35: 1590-1592, 1994.

4. Saleh M and Trinchieri G: Innate immune mechanisms of colitis and colitis-associated colorectal cancer. Nat Rev Immunol 11: 9-20, 2011.

5. Rhodes JM: Unifying hypothesis for inflammatory bowel disease and associated colon cancer: sticking the pieces together with sugar. Lancet 347: 40-44, 1996. 
6. Rutter M, Saunders B, Wilkinson K, et al: Severity of inflammation is a risk factor for colorectal neoplasia in ulcerative colitis. Gastroenterology 126: 451-459, 2004.

7. Strimpakos AS, Karapanagiotou EM, Saif MW and Syrigos KN: The role of mTOR in the management of solid tumors: an overview. Cancer Treat Rev 35: 148-159, 2009.

8. Guertin DA and Sabatini DM: Defining the role of mTOR in cancer. Cancer Cell 12: 9-22, 2007.

9. Hay N and Sonenberg N: Upstream and downstream of mTOR. Genes Dev 18: 1926-1945, 2004

10. Delgoffe GM and Powell JD: mTOR: taking cues from the immune microenvironment. Immunology 127: 459-465, 2009.

11. Magnuson B, Ekim B and Fingar DC: Regulation and function of ribosomal protein S6 kinase (S6K) within mTOR signalling networks. Biochem J 441: 1-21, 2012.

12. Jastrzebski K, Hannan KM, Tchoubrieva EB, Hannan RD and Pearson RB: Coordinate regulation of ribosome biogenesis and function by the ribosomal protein S6 kinase, a key mediator of mTOR function. Growth Factors 25: 209-226, 2007.

13. Duvel K, Yecies JL, Menon S, et al: Activation of a metabolic gene regulatory network downstream of mTOR complex 1 . Mol Cell 39: 171-183, 2010.

14. Guertin DA and Sabatini DM: An expanding role for mTOR in cancer. Trends Mol Med 11: 353-361, 2005.

15. Sarbassov DD, Ali SM, Sengupta S, et al: Prolonged rapamycin treatment inhibits mTORC2 assembly and Akt/PKB. Mol Cell 22: 159-168, 2006.

16. Bjornsti MA and Houghton PJ: The TOR pathway: a target for cancer therapy. Nat Rev Cancer 4: 335-348, 2004.

17. Thiem S, Pierce TP, Palmieri M, et al: mTORC1 inhibition restricts inflammation-associated gastrointestinal tumorigenesis in mice. J Clin Invest 123: 767-781, 2013.

18. Yu H, Pardoll D and Jove R: STATs in cancer inflammation and immunity: a leading role for STAT3. Nat Rev Cancer 9: 798-809, 2009.

19. Grivennikov S, Karin E, Terzic J, et al: IL-6 and Stat3 are required for survival of intestinal epithelial cells and development of colitis-associated cancer. Cancer Cell 15: 103-113, 2009.

20. Chumanevich AA, Poudyal D, Cui X, et al: Suppression of colitisdriven colon cancer in mice by a novel small molecule inhibitor of sphingosine kinase. Carcinogenesis 31: 1787-1793, 2010.

21. Namba R, Young LJ, Abbey CK, et al: Rapamycin inhibits growth of premalignant and malignant mammary lesions in a mouse model of ductal carcinoma in situ. Clin Cancer Res 12: 2613-2621, 2006

22. Yu H, Kortylewski M and Pardoll D: Crosstalk between cancer and immune cells: role of STAT3 in the tumour microenvironment. Nat Rev Immunol 7: 41-51, 2007.

23. Park E, Park J, Han SW, et al: NVP-BKM120, a novel PI3K inhibitor, shows synergism with a STAT3 inhibitor in human gastric cancer cells harboring KRAS mutations. Int J Oncol 40: 1259-1266, 2012.

24. Farraye FA, Odze RD, Eaden J and Itzkowitz SH: AGA technical review on the diagnosis and management of colorectal neoplasia in inflammatory bowel disease. Gastroenterology 138: 746-774.e4, 2010.

25. Gupta RB, Harpaz N, Itzkowitz S, et al: Histologic inflammation is a risk factor for progression to colorectal neoplasia in ulcerative colitis: a cohort study. Gastroenterology 133: 1099-1105, 2007.

26. Sobala A, Herbst F, Novacek G and Vogelsang H: Colorectal carcinoma and preceding fistula in Crohn's disease. J Crohns Colitis 4: 189-193, 2010.

27. Laukoetter MG, Mennigen R, Hannig CM, et al: Intestinal cancer risk in Crohn's disease: a meta-analysis. J Gastrointest Surg 15 576-583, 2011.
28. van Staa TP, Card T, Logan RF and Leufkens HG: 5-Aminosalicylate use and colorectal cancer risk in inflammatory bowel disease: a large epidemiological study. Gut 54: 1573-1578, 2005

29. Rubin DT, LoSavio A, Yadron N, Huo D and Hanauer SB Aminosalicylate therapy in the prevention of dysplasia and colorectal cancer in ulcerative colitis. Clin Gastroenterol Hepatol 4: 1346-1350, 2006.

30. Slomovitz BM and Coleman RL: The PI3K/AKT/mTOR pathway as a therapeutic target in endometrial cancer. Clin Cancer Res 18: 5856-5864, 2012.

31. Lauring J,Park BH and Wolff AC: The phosphoinositide-3-kinaseAkt-mTOR pathway as a therapeutic target in breast cancer. J Natl Compr Cancer Netw 11: 670-678, 2013.

32. Lee YK, Park SY, Kim YM, et al: Suppression of mTOR via Akt-dependent and -independent mechanisms in selenium-treated colon cancer cells: involvement of AMPK $\alpha 1$. Carcinogenesis 31: 1092-1099, 2010

33. Zhang DM, Liu JS, Deng LJ, et al: Arenobufagin, a natural bufadienolide from toad venom, induces apoptosis and autophagy in human hepatocellular carcinoma cells through inhibition of PI3K/Akt/mTOR pathway. Carcinogenesis 34: 1331-1342, 2013.

34. Smrz D, Kim MS, Zhang S, et al: mTORC1 and mTORC2 differentially regulate homeostasis of neoplastic and non-neoplastic human mast cells. Blood 118: 6803-6813, 2011.

35. Fox R, Nhan TQ, Law GL, Morris DR,Liles WC and Schwartz SM: PSGL-1 and mTOR regulate translation of ROCK-1 and physiological functions of macrophages. EMBO J 26: 505-515, 2007.

36. Kawauchi K, Ihjima K and Yamada O: IL-2 increases human telomerase reverse transcriptase activity transcriptionally and posttranslationally through phosphatidylinositol 3'-kinase/Akt, heat shock protein 90 , and mammalian target of rapamycin in transformed NK cells. J Immunol 174: 5261-5269, 2005.

37. Donahue AC and Fruman DA: Proliferation and survival of activated $B$ cells requires sustained antigen receptor engagement and phosphoinositide 3-kinase activation. J Immunol 170: 5851-5860, 2003

38. Powell JD and Delgoffe GM: The mammalian target of rapamycin: linking $\mathrm{T}$ cell differentiation, function, and metabolism. Immunity 33: 301-311, 2010.

39. Memmott RM and Dennis PA: The role of the Akt/mTOR pathway in tobacco carcinogen-induced lung tumorigenesis. Clin Cancer Res 16: 4-10, 2010.

40. Ma J, Meng Y, Kwiatkowski DJ, et al: Mammalian target of rapamycin regulates murine and human cell differentiation through STAT3/p63/Jagged/Notch cascade. J Clin Invest 120: 103-114, 2010.

41. Farkas S, Hornung M, Sattler C, et al: Rapamycin decreases leukocyte migration in vivo and effectively reduces experimentally induced chronic colitis. Int J Colorectal Dis 21: 747-753, 2006.

42. Deng L, Zhou JF, Sellers RS, et al: A novel mouse model of inflammatory bowel disease links mammalian target of rapamycin-dependent hyperproliferation of colonic epithelium to inflammation-associated tumorigenesis. Am J Pathol 176: 952-967, 2010

43. Wu Y and Zhou BP: TNF- $\alpha / \mathrm{NF}-\kappa \mathrm{B} /$ Snail pathway in cancer cell migration and invasion. Br J Cancer 102: 639-644, 2010.

44. Hodge DR, Hurt EM and Farrar WL: The role of IL-6 and STAT3 in inflammation and cancer. Eur J Cancer 41: 2502-2512, 2005.

45. Grivennikov S, Karin E, Terzic J, et al: IL-6 and Stat3 are required for survival of intestinal epithelial cells and development of colitis-associated cancer. Cancer Cell 15: 103-113, 2009.

46. Lippitz BE: Cytokine patterns in patients with cancer: a systematic review. Lancet Oncol 14: e218-e228, 2013. 